\title{
Prevalence and expression of photosensitivity in systemic lupus erythematosus
}

\author{
A J WYSENBEEK, D A BLOCK, AND J F FRIES \\ From the Department of Medicine, Stanford University School of Medicine, Stanford, California, USA
}

SUMMARY Photosensitivity was assessed in 125 patients with systemic lupus erythematosus (SLE) and in 281 patients with rheumatoid arthritis (RA) as controls. Photosensitivity was reported by $87 / 119(73 \%)$ patients with SLE and in $62 / 269(23 \%)$ patients with RA; involving the face in $72 / 122(59 \%)$ patients with SLE, then arms, chest, and neck. Patients with SLE reported that sun exposure could exacerbate various systemic symptoms, 51/121 (42\%) reported medical treatment for photosensitivity and 41/118 (35\%) reported that photosensitivity had a significant impact on their lifestyle. There was no significant difference in disease severity, as judged by physician or laboratory results, between patients scoring high or low on the photosensitivity scale.

Patients with systemic lupus erythematosus (SLE) are known often to be sensitive to sun exposure. ${ }^{12}$ Although information about the prevalence of photosensitivity has been reported, ${ }^{3-12}$ this information is not current and there are few data about the expression and impact of photosensitivity and its relation to systemic, non-cutaneous disease.

We presented a photosensitivity questionnaire to a group of patients with SLE and a control group of patients with rheumatoid arthritis (RA). Then, to investigate further the relation between photosensitivity and SLE disease activity we examined the relation between the photosensitivity score and various SLE variables obtained from the ARAMIS (American Rheumatism Association Medical Information System) data bank.

\section{Patients and methods}

PATIENTS AND CONTROLS

Consecutive patients with SLE and RA from Stanford University and Johns Hopkins University were assessed by questionnaires mailed biannually as part of routine outcome studies conducted by ARAMIS.

PHOTOSENSITIVITY QUESTIONNAIRE

This questionnaire assessed the degree of photosensitivity on a visual analogue scale, dermal and

Accepted for publication 6 October 1988.

Correspondence to Dr A J Wysenbeek, Department of Medicine B and the Rheumatology Unit, Beilinson Medical Center, 49100 Petah Tiqva, Israel. systemic reactions to sun exposure, chronological relation of photosensitivity to disease initiation, and its impact on medical treatment and lifestyle.

RELATION BETWEEN PHOTOSENSITIVITY AND DISEASE EXPRESSION

Patients with SLE were divided into two subgroups-those scoring below or above the SLE mean score on the photosensitivity analogue scale. These two subgroups were compared by 47 ARAMIS variables divided into three main categories: $(a)$ patients' self reported symptoms; $(b)$ physicians' assessments; $(c)$ laboratory tests. For patients with multiple recordings of a variable the last recorded value was used.

Statistical analysis was by two tailed Student's $t$ test, two way Wilcoxon's test, and $\chi^{2}$ test for interval, ordinal, or nominal distribution of data respectively. Correlation was by Pearson's product moment correlation coefficient.

\section{Results}

The photosensitivity questionnaire return was $77.6 \%$ for the SLE group and $83.8 \%$ for the RA group. There was no difference in background data for patients with SLE and RA who did, or did not, return the questionnaire, or between Stanford and Johns Hopkins data banks.

The study included 125 patients with SLE, mean age 42.5 (SD 13.4) years and 281 patients with RA, mean age $55.8(13.8)$ years. Age had no correlation 
with photosensitivity $(r=0.01$ for SLE, $r=0.03$ for RA). The patients with SLE had dark complexions, hair, and eyes. Patients with light complexion, hair, or eyes scored higher on the photosensitivity scale (data not shown).

Patients with SLE scored an average of 1.9 (SD 0.9 ) out of 3.0 on the photosensitivity scale, while patients with RA scored $1.2 \quad(0.9) \quad(p<0.0001)$. Eighty seven out of $119(73 \%)$ patients with SLE and $62 / 269(23 \%)$ patients with RA scored above 1.5 on the scale.

Tables 1 and 2 show the skin areas and systemic reactions reported to be involved in photosensitive reactions. Table 3 shows the effect of photosensitivity on lifestyle.

Twenty nine per cent of patients with SLE reported use of corticosteroid cream, $22 \%$ use of

Table 1 Skin areas in which a rash developed or worsened after sun exposure. Figures show number (\%) of patients

\begin{tabular}{llll}
\hline Skin area & $\begin{array}{l}S L E^{*} \\
(n=122)\end{array}$ & $\begin{array}{l}R A^{*} \\
(n=269)\end{array}$ & $p$ Value \\
\hline Face & $72(59)$ & $40(15)$ & $<0.0001$ \\
Arms & $57(47)$ & $49(18)$ & $<0.0001$ \\
Upper chest & $44(36)$ & $37(14)$ & $<0.0001$ \\
Neck & $37(30)$ & $28(10)$ & $<0.0001$ \\
Back & $30(25)$ & $13(5)$ & $<0.0001$ \\
Other & $22(18)$ & $14(5)$ & 0.0001 \\
\hline
\end{tabular}

*SLE=systemic lupus erythematosus; RA=rheumatoid arthritis.

Table 2 Reported induction or increase of systemic complaints after sun exposure. Figures show number (\%) of patients

\begin{tabular}{lllr}
\hline $\begin{array}{l}\text { Systemic } \\
\text { complaint }\end{array}$ & $\begin{array}{l}S L E^{*} \\
(n=120)\end{array}$ & $\begin{array}{l}R A^{*} \\
(n=268)\end{array}$ & $p$ Value \\
\hline $\begin{array}{l}\text { Weakness, } \\
\text { fatigue }\end{array}$ & $89(74)$ & $99(37)$ & $<0.0001$ \\
$\begin{array}{l}\text { Joint pain } \\
\text { Rash to }\end{array}$ & $47(39)$ & $20(7)$ & $<0.0001$ \\
$\quad \begin{array}{l}\text { unexposed skin } \\
\text { Fever }\end{array}$ & $38(32)$ & $10(4)$ & $<0.0001$ \\
Hair loss & $33(28)$ & $13(5)$ & $<0.0001$ \\
Other & $14(12)$ & $7(3)$ & 0.0007 \\
\hline
\end{tabular}

*SLE=systemic lupus erythematosus; RA=rheumatoid arthritis.

Table 3 Effect of photosensitivity on patients' lifestyle.* Figures show number (\%) of patients

\begin{tabular}{lll}
\hline & $\begin{array}{l}S L E^{+} \\
(n=118)\end{array}$ & $\begin{array}{l}R A^{+} \\
(n=262)\end{array}$ \\
\hline Significant change & $41(35)$ & $14(5)$ \\
Minor change & $47(40)$ & $82(31)$ \\
No change & $20(17)$ & $80(31)$ \\
Not sensitive to sun & $10(8)$ & $86(33)$ \\
\hline
\end{tabular}

*p<0.0001.

+SLE=systemic lupus erythematosus; $R A=$ rheumatoid arthritis. antimalarial drugs, and $21 \%$ increase of steroides? dose, all owing to photosensitivity. Forty two per cent of the patients with SLE entered a positive reply for one or more of the possible treatments.

Of the 47 ARAMIS variables examined for $a \overline{0}$ possible relation to photosensitivity, for physician? assessment, and for laboratory tests, the distribution was relatively even between more pathologicaps variables in the SLE photosensitive and nonphotosensitive groups. Thus platelets were lower in the photosensitive group $\left(247(88) \times 10^{9} / 1 \quad v 291 \vec{\omega}\right.$ $\left.(93) \times 10^{9} / 1 ; p=0.04\right)$ and urine protein $(0-4)$ higher in the non-photosensitive group $(0.54(1.0) \vee 1.08$ $(1.4) ; p=0.06)$. Of patients' self reported symptoms the photosensitive group had a higher pathologica score for global arthritis assessment $(32 v 18$ $\mathrm{p}=0.02)$, mouth ulcers $(59 \% \vee 32 \% ; \mathrm{p}=0.01)$, and muscle pain $(42 \% v 17 \% ; \mathrm{p}=0.017)$. All differences between the two subgroups of patients with SLE loststatistical significance after correction for multiple comparisons.

\section{Discussion}

This study gathered information about the prevalence and expression of photosensitivity in patients with SLE. Previous studies have reported photosen-o sitivity in patients with $\mathrm{SLE}^{3-12}$ ranging from $32.7 \%$ of Dubois' patients ${ }^{9}$ to $43 \%$ of patients in the 19820 revised criteria for SLE. ${ }^{12}$

We differentiated between cutaneous andB systemic symptoms secondary to sun exposure Patients with SLE described photosensitivity over. face, arms, chest, neck, and back in descendingo order, while patients with RA described involve? ment of these areas with a low, relatively equa? frequency. In contrast with patients with SLE, the patients with RA rarely reported systemic symptoms, except weakness and fatigue. Photosensitivity also caused significant changes in lifestyle and medica $B$ treatment of patients with SLE.

Patients with SLE reported that several disease symptoms were increased by sun exposure. Our ex N amination of various data bank variables, however did not show increased disease expression in the photosensitive group according to physician assess $-\omega$ ment or in laboratory variables but only a tendency? towards a higher score for self reported variables Thus photosensitive patients with SLE reporti associated systemic problems, but these do not appear to be related to standard laboratory assess- $-\mathrm{C}$ ments of severity. Although sun exposure may increase systemic disease symptoms in individual patients with SLE, we were unable to show that this phenomenon is related to more severe overallo disease expression. 
Large fractions of both study $(87 / 119,73 \%)$ and control $(62 / 269,23 \%)$ groups were in the upper half of the self reported photosensitivity scale. This may be explained by the public awareness in general, and in patients with SLE in particular, of potential ultraviolet induced hazards. We also found that $51 / 121(42 \%)$ patients with SLE reported some change in their medical treatment owing to photosensitivity. Thus the prevalence of photosensitivity in SLE may be somewhere between $42 \%$ and $73 \%$. This percentage is actually less important than the observation that apparent increased awareness of potential ultraviolet damage is associated with very significant changes in the lifestyle of patients with SLE.

We thank John Oehlert and Dee Simpson for assisting in computerised data handling, Patricia Spitz for reviewing the manuscript, and Dr Marc Hochberg for providing access to the Johns Hopkins data. Supported by NIH grant AR-21393 to the American Rheumatism Association Medical Information System (ARAMIS).

\section{References}

1 Rothfield N F. Clinical features of systemic lupus erythemato- sus. In: Kelly W N, Harris E D, Ruddy S, Sledge C D, eds. Textbook of rheumatology. 2nd ed. Philadelphia: Saunders, 1985: 1077.

2 Rothfield N F. Systemic lupus erythematosus: clinical aspects and treatment. In: McCarty D J, ed. Arthritis and allied conditions. 10th ed. Philadelphia: Lea and Febiger, 1985: 912.

3 Epstein J H. Tuffanelli D L. Dubois E L. Light sensitivity and lupus erythematosus. Arch Dermatol 1965; 91: 483-5.

4 Baer R L. Harber L C. Photobiology of lupus erythematosus. Arch Dermatol 1965; 92: 124-8.

5 Everett M A, Olson R L. Response of cutaneous lupus erythematosus to ultraviolet light. $J$ Invest Dermatol 1965; 44: 133-4.

6 Pollock B H, Stevens M B. Shulman L E. Photosensitivity in lupus erythematosus. Arthritis Rheum 1966; 9: 533.

7 Freeman R G, Knox J M, Owens D W. Cutaneous lesions of lupus erythematosus induced by monochromatic light. Arch Dermatol 1969; 100: 677-82.

8 Cripps D J, Rankin J. Action spectra of lupus erythematosus and experimental immunofluorescence. Arch Dermatol 1973: 107: 563-7.

9 Dubois E L. Lupus erythematosus. 2nd ed. Los Angeles: University of Southern California Press, 1974: 289-91.

10 Ropes M W. Systemic lupus erythematosus. Cambridge: Harvard University Press, 1976: 22-3.

11 Rothfield N F. Lupus erythematosus. In: Fitzpatrick T B, Eisen A, Wolff K, Freedberg I M. Austen K F, eds. Dermatology in general medicine. 2nd ed. New York: McGraw-Hill: 1979: 1281.

12 Tan E M, Cohen A S. Fries J F, et al. The 1982 revised criteria for the classification of systemic lupus erythematosus. Arthritis Rheum 1982; 25: 1271-7. 